\title{
THE THERMAL CASIMIR EFFECT: SATURATION
}

\author{
Bo E Sernelius
}

\section{Linköping University Post Print}

N.B.: When citing this work, cite the original article.

Electronic version of an article published as:

Bo E Sernelius , THE THERMAL CASIMIR EFFECT: SATURATION, 2010, INTERNATIONAL JOURNAL OF MODERN PHYSICS A, (25), 11, 2319-2327. http://dx.doi.org/10.1142/S0217751X10049591

Copyright: World Scientific Publishing http://www.worldscinet.com/

Postprint available at: Linköping University Electronic Press http://urn.kb.se/resolve?urn=urn:nbn:se:liu:diva-56685 


\title{
THE THERMAL CASIMIR EFFECT: SATURATION
}

\author{
Bo E. Sernelius \\ Division of Theory and Modeling, Department of Physics, Chemistry and Biology, \\ Linköping University, SE-581 83 Linköping, Sweden \\ *E-mail: bos@ifm.liu.se
}

This article addresses the discrepancies between theoretical and experimental results obtained for the thermal Casimir effect. Here we test the possibility that saturation effects may be the root of the problems. We present graphs that describe the numerical derivations in great detail.

Keywords: Casimir; thermal effects; dissipation; saturation; massless bosons

\section{Introduction}

We realized ${ }^{1}$ that the correct way to treat the materials in the Casimir experiments is to include finite temperature, finite conductivity and dissipation, all at the same time. Up till then one had started from perfect metals and added corrections, one by one, for the effects just mentioned. Very soon it became evident that this more realistic treatment of the materials led to worse agreement with the experimental results. This divided the Casimir community into two groups: one in favor of the more realistic treatment based on known material properties; one in favor of a theory that agrees better with experiment but, for this to be the case, has to invoke various prescriptions - a phenomenological approach. The general situation is one of great frustration. The consensus in the Casimir community at present is that the correct approach is to use the proper material properties and that both experimentalists and theorists should do their best in finding the root of the problem.

In the present work we test if the cause of all the problems can be saturation effects. The electromagnetic normal modes involved in the Casimir effect are assumed to be independent massless bosons. However, these bosons are formed from electron excitations and the electrons are fermions. This means that the modes are not completely independent. Saturation effects are expected to appear in case a huge number of modes are excited. We 
test this idea on several different Casimir experiments where theory and experiment disagree. First out is the classical Casimir force measurement between two metal half spaces; here both in the form of the torsion pendulum experiment by Lamoreaux ${ }^{2}$ and in the form of the Casimir pressure measurement between a gold sphere and a gold plate as performed by Decca et al. $^{3}$; theory predicts a large negative thermal correction, absent in the high precision experiments. The third experiment is the measurement of the Casimir force between a metal plate and a laser irradiated semiconductor membrane as performed by Chen et al. $^{4}$; the change in force with laser intensity is larger than predicted by theory. The fourth experiment is the measurement of the Casimir force between an atom and a wall in the form of the measurement by Obrecht et al. ${ }^{5}$ of the change in oscillation frequency of a ${ }^{87} R b$ Bose-Einstein condensate (BEC) trapped to a fused silica wall; the change is smaller than predicted by theory.

The main results from the work is to be presented elsewhere. ${ }^{6,7}$ To avoid too much overlap we here make a much briefer description of the formal derivation and expand more on where the cause of the deviations are to be found and what the effects are from our saturation corrections. In Sec. 2 we summarize the formalism; in Sec. 3 we discuss the experiments by Lamoreaux and Decca; Sec. 4 is devoted to the experiment by Chen et al.; in Sec. 5 we discuss the experiment by Obrecht et al.; Sec. 6 contains a brief summary.

\section{Basic Formalism}

The theory corresponding to all experiments treated here are based on the two plate geometry. Even the Casimir force between an atom and a wall can be obtained from the results of this geometry; one takes the limit when the thickness of one of the plates goes to zero and at the same time lets the material of the thin plate be diluted. In the two plate geometry the interaction energy per unit area, $V(d)$, can at zero temperature be written on the form ${ }^{8}$

$$
V(d)=\frac{\hbar}{\Omega} \sum_{\mathbf{k}} \int_{0}^{\infty} \frac{d \omega}{2 \pi} \ln [f(\mathbf{k}, i \omega)],
$$

where $d$ is the distance between the plates, $\mathbf{k}$ is the two-dimensional wave vector in the plane of the plates, $\Omega$ is the area of a plate, and $f(\mathbf{k}, \omega)=0$ is the condition for an electromagnetic normal mode in the particular geometry. This expression one arrives at using the extended argument principle ${ }^{8}$ 


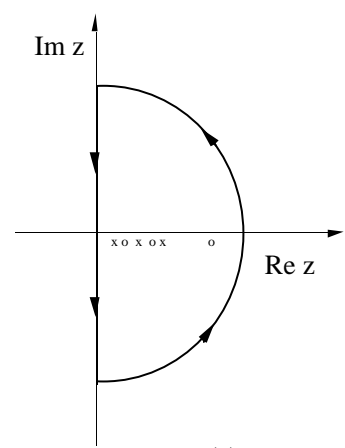

(a)

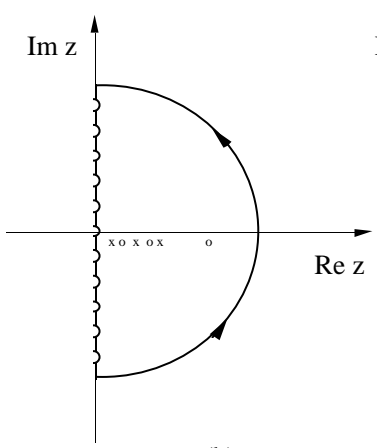

(b)

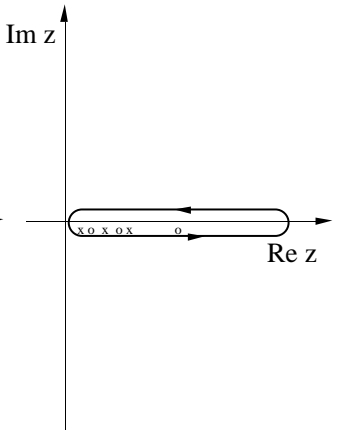

(c)

Fig. 1. Integration contours in the complex frequency plane used in connection with the extended argument principle: (a) contour used for zero temperature; (b) contour used for finite temperature; (c) contour that can be used both at zero and finite temperature.

with the contour shown in Fig. 1(a). At finite temperature the integration is replaced by a discrete summation over Matsubara frequencies,

$$
V(d)=\frac{1}{\beta \Omega} \sum_{\mathbf{k}} \sum_{\omega_{n}}^{\prime} \ln \left[f\left(\mathbf{k}, i \omega_{n}\right)\right] ; \omega_{n}=\frac{2 \pi n}{\hbar \beta} .
$$

This expression one arrives at using the contour shown in Fig. 1(b). Alternatively one may use the contour shown in Fig. 1(c) and integrate along the real frequency axis,

$$
V(d)=\frac{2 \hbar}{\Omega} \sum_{\mathbf{k}} \operatorname{Im} \int_{0}^{\infty} \frac{d \omega}{2 \pi}[n(\omega)+1 / 2] \ln [f(\mathbf{k}, \omega)],
$$

where $n(\omega)=[\exp (\hbar \beta \omega)-1]^{-1}$ is the distribution function for massless bosons. This form can also be used at zero temperature; then the distribution function vanishes. Problems encountered using the real frequency integration is discussed in detail elsewhere. ${ }^{9}$ In the two plate geometry there are two groups of normal mode, transverse magnetic (TM) and transverse electric (TE), each with a different mode condition function. The interaction potential is a sum of two terms, $V(d)=V^{T M}(d)+V^{T E}(d)$.

The distribution function in Eq. (3) diverges for zero frequency and it is the low energy modes that cause the problems in all experiments treated here. We shift the distribution function downwards in frequency, so that it never reaches the point of divergence, by adding a damping parameter, $D$,

$$
\tilde{n}(\omega)=[\exp (\hbar \beta \omega+D)-1]^{-1} .
$$




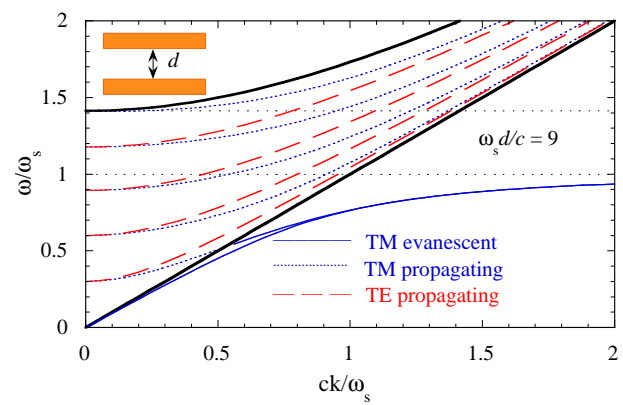

Fig. 2. Dispersion curves for the modes between two gold plates in absence of dissipation. The frequencies are in units of $\omega_{s}$, the surface plasmon frequency. The solid straight line is the light dispersion curve in vacuum; the dashed (dotted) curves are TE (TM) propagating modes; the thin solid curves are evanescent TM modes; the thick solid curve is the lower boundary for transverse bulk modes in the plates. From Ref. [10].

The discrete frequency summation in Eq. (2) is the result of the poles of the distribution function that all fall on the imaginary axis. ${ }^{8}$ Our new distribution function has its poles shifted away from the axis the distance $D / \hbar \beta$ into the left half plane. The new form of the interaction potential is

$$
V(d)=\frac{1}{\beta \Omega} \sum_{\mathbf{k}} \sum_{\omega_{n}} \frac{1}{\pi} \int_{-\infty}^{\infty} d \omega^{\prime} \frac{(D / \beta) \ln \left[f\left(k, i \omega^{\prime}\right)\right]}{\left(\omega^{\prime}-\omega_{n}\right)^{2}+(D / \beta)^{2}} .
$$

Each term in the original summation is replaced by an integral. For small $D$ values it is enough to replace only the zero frequency term.

\section{Two Parallel Metal Plates}

This geometry is applicable to the torsion pendulum experiment by Lamoreaux $^{2}$ and the Casimir pressure measurement performed by Decca et al. ${ }^{3}$ The dispersion curves for the electromagnetic normal modes for two gold plates $^{10}$ are shown in Fig. 2. This figure is valid in neglect of dissipation in the plate materials. The modes are propagating (evanescent) above and to the left (below and to the right) of the light dispersion curve. The light dispersion curve is the straight diagonal line in the figure; it has slope unity with the chosen scaling of the axes. Note that there are no TE evanescent modes. When the system is allowed to have dissipation there are modes everywhere. Each original mode is replaced by a continuum of modes. ${ }^{11}$ Evanescent TE modes appear and the continuum extends all the way down to the momentum axis. These modes are the cause of all the problems with 


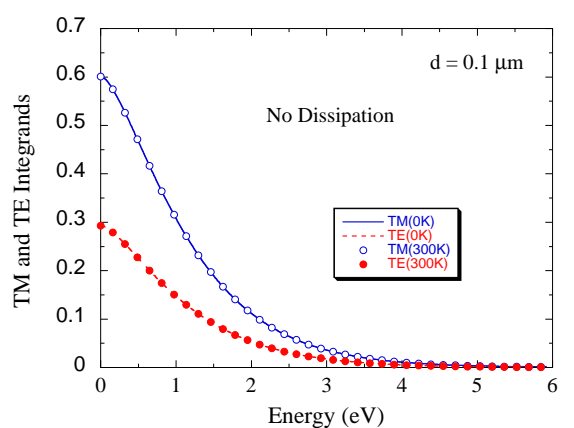

(a)

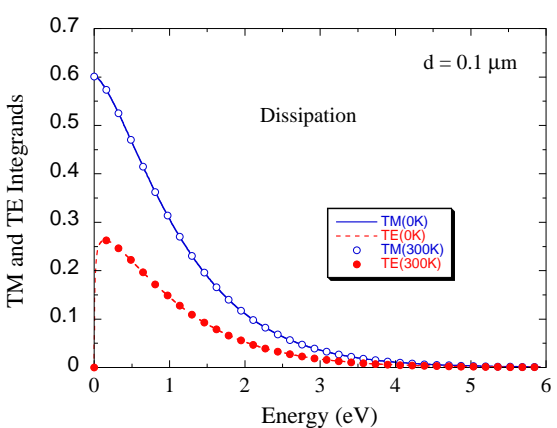

(b)

Fig. 3. Integrands of Eq. (1) (curves) and summands of Eq. (2) (circles) in the case of two gold plates separated by $0.1 \mu \mathrm{m}$.

the thermal Casimir force in this geometry. The integrand of Eq. (1) and

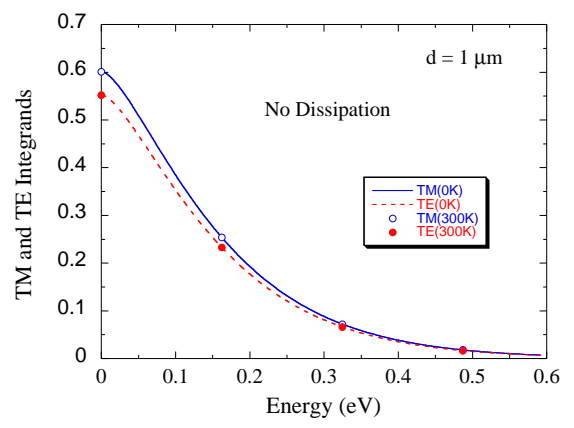

(a)

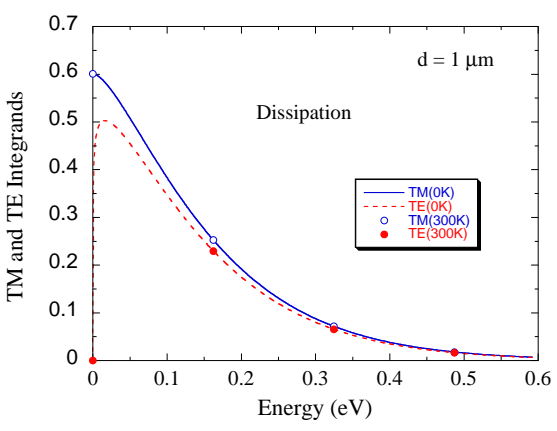

(b)

Fig. 4. Same as Fig. 3 but now for $1.0 \mu m$.

summand of Eq. (2) in the case of two gold plates separated by $0.1 \mu \mathrm{m}$ are displayed in Fig. 3. Fig. 3(a) is the result when dissipation is neglected and Fig. 3(b) when dissipation is included. The solid (dashed) curves is the TM (TE) integrand; the open (filled) circles is the TM (TE) summand. We note that the effect of dissipation is to push down the TE integrand toward zero for small energies or frequencies. In Eq. (2) the $n=0$ term vanishes for the TE contribution. We further note that for this separation the circles come closely spaced and the summation and integration give very similar results - there are very small temperature effects; the integrand is pushed down very close to the vertical axis - the dissipation has a very small effect on the zero temperature results. 


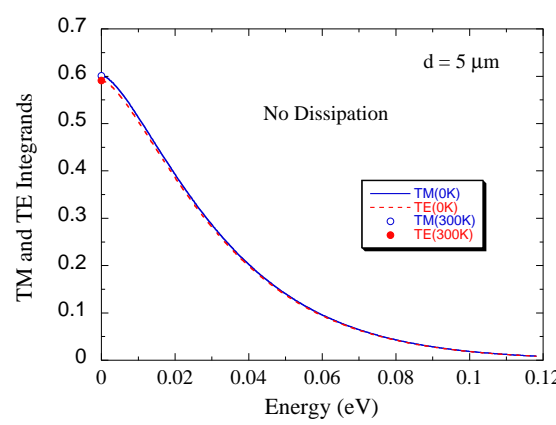

(a)

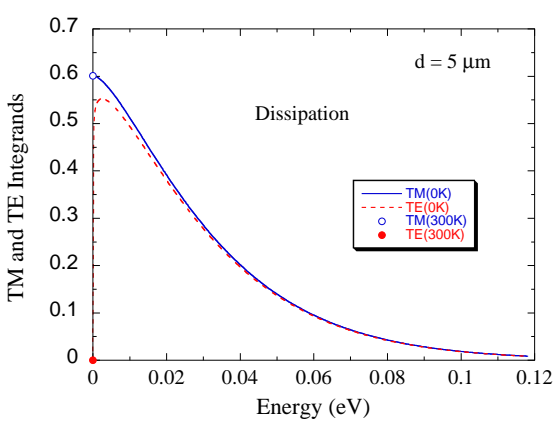

Fig. 5. Same as Fig. 3 but now for $5 \mu m$.

In Fig. 4 we show the results at $1.0 \mu m$ separation. Here we note that summation points are much fever and hence the temperature effect is much larger; the dissipation has still negligible effect on the zero temperature result; on the room temperature result, on the other hand, it has a huge effect - the TE contribution has been reduced by a factor of two. For $5 \mu \mathrm{m}$ separation, Fig. 5, we note that only one summation point contributes; the dissipation has the effect that at room temperature there is nolonger any TE contribution at all.

In Fig. 6(a) we show the energy correction factor for the two-gold-plate geometry. The energy correction factor is the Casimir energy per unit area divided by the zero temperature Casimir energy per unit area for two perfectly reflecting metal plates, $-\hbar c \pi^{2} /\left(720 d^{3}\right)$. The experimental results ${ }^{2}$ obtained by Lamoreaux are shown as squares with error bars. There is a cluster of experimental points near $1 \mu \mathrm{m}$ separation. Here the deviation between theory and experiment is over $20 \%$. Theory and experiment are in clear disagreement. The experimental result ${ }^{3}$ for the normalized Casimir pressure at $295 \mathrm{~K}$ is shown as dots in Fig. 6(b). The bars are the endpoints of the experimental error bars. The upper (lower) thick solid curve is the theoretical result for zero temperature $(295 \mathrm{~K})$ calculated with Eqs. (1) and (2), respectively. The dielectric function on the imaginary frequency axis was derived from experimental tabulated optical data for gold. We note that the zero temperature result agrees much better with the experimental result. The large negative thermal correction comes entirely from the TE evanescent modes. All curves are normalized with the zero temperature Casimir pressure between two perfectly reflecting metal plates, $\hbar c \pi^{2} /\left(240 d^{4}\right)$. The circles are the results for different damping parame- 


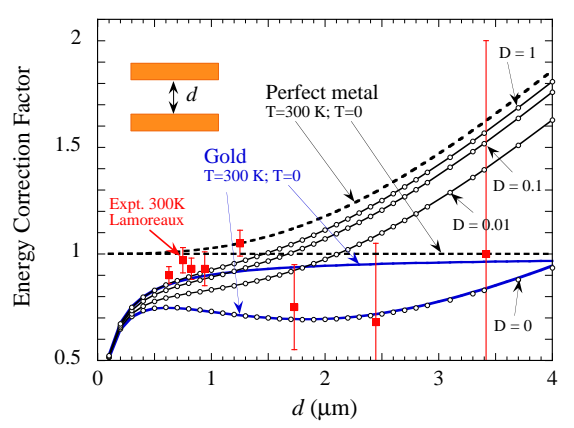

(a)

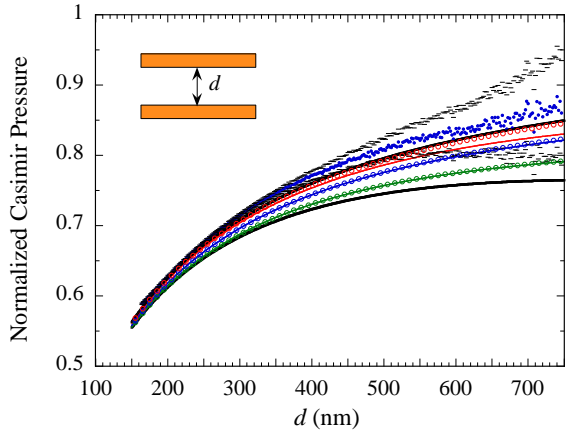

(b)

Fig. 6. (a): Energy correction factor for two gold plates. The filled squares with error bars are the Lamoreaux' experimental ${ }^{2}$ values from the torsion pendulum experiment. The dashed curves are the perfect metal results. The thick solid curves are the results for real gold plates using Eq. (1) for zero temperature and Eq. (2) for room temperature. The curves with circles are the results from our model calculations with different saturation parameters; (b): Casimir pressure between two gold plates. The experimental result ${ }^{3}$ is shown as dots and the endpoints of the error bars are indicated by horizontal bars; the upper (lower) thick solid curve is the traditional theoretical zero (room) temperature result; the circles are the present results, obtained from Eq. (3) with the distribution function for TE evanescent waves modified according to Eq. (4), with damping parameters $0.01,0.1$, and 1.0 , respectively, counting from below; the corresponding results obtained by shifting the zero frequency pole into the left of the complex frequency plane are shown as thin solid curves.

ters from using Eq. (3) with the modified distribution function of Eq. (4) in the contribution from the TE evanescent waves. To each set of circles corresponds a thin solid curve. This curve is the result of using Eq. (2), where just the zero frequency pole has been moved into the left half of the complex frequency plane and the corresponding term in the summation has been modified according to Eq. (5). We note that for the two lowest set of curves with small damping the two results agree. For very high damping there are deviations. These deviations have two reasons: One is that in the thin solid curves all mode types are affected by the damping; the second is that for strong enough damping more terms in the summation should be modified. In Fig. 7(a) we show the thermal correction to the Casimir energy. The contribution from all four mode types are given separately. These curves have been obtained from Eq. (3) and the Drude dielectric function suited for gold has been used. We note that the TE evanescent waves give a negative contribution. For larger separations the total TE contribution saturates when it has completely eliminated the zero temperature TE contribution. Fig. 7(b) shows how the integrand for the TE evanescent modes 


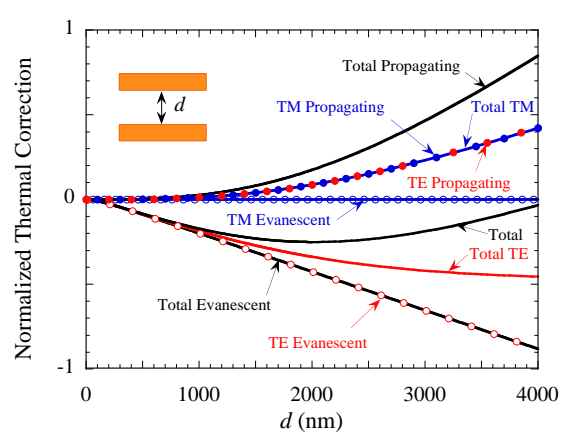

(a)

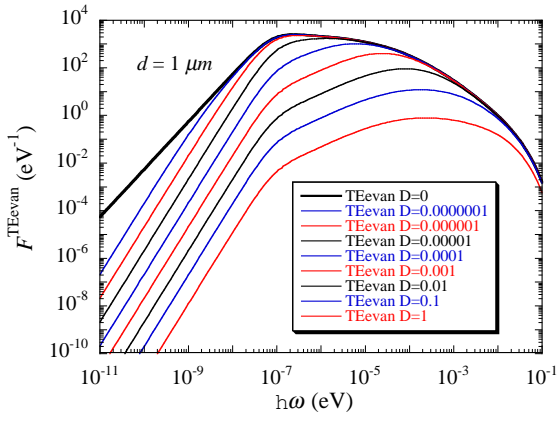

(b)

Fig. 7. (a): The thermal contribution to the Casimir energy from the four mode types between two gold plates. The results are also lumped together in various ways. All results are divided by the zero temperature Casimir energy for perfectly reflecting plates; (b): The integrand for the TE evanescent modes for different choices of damping parameter.

is affected by the choice of damping parameter.

\section{Two Parallel Plates, one Metallic and one Semiconducting}

The third experiment we consider here is the measurement of the Casimir force between a gold plate and a laser irradiated semiconductor membrane as performed by Chen et al. ${ }^{4}$ They measured the change in force with the laser irradiation compared to without any irradiation. The idea is to find out how the force varies with carrier concentration in the semiconducting membrane. The results are shown in Fig. 8(a). The open squares with error bars are the experimental result. The dashed curve with open circles is the theoretical result for $300 \mathrm{~K}$. The deviations are clear. In this geometry it is not enough to neglect dissipation to get agreement with experiment; the theoretical results with and without dissipation are very similar. Besides, it is now the TM modes that cause the problems. The solid curve with filled (open) circles is our saturation based result with $D$ equal to 0.01 (0.1). We find that both these curves agree with the experiment within the experimental uncertainty. Here we have used Eq. (2) and just modified the zero frequency contribution according to Eq. (5).

\section{Atom Wall Geometry}

In the experiment by Obrecht et al. ${ }^{5}$ one studied indirectly the force between a rubidium atom and a dielectric substrate. This was done by mea- 


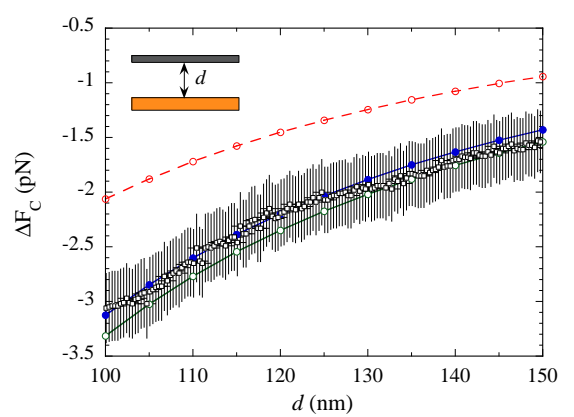

(a)

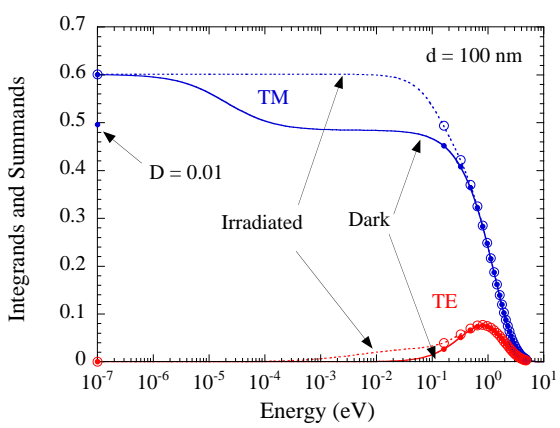

(b)

Fig. 8. (a): The change in Casimir force, at $300 \mathrm{~K}$, between a gold sphere and a silicon membrane with and without laser irradiation. The open squares with error bars are the experimental ${ }^{4}$ result. The dashed curve with open circles is the theoretical result without saturation effects. The solid curve with filled (open) circles is our present result with $D$ equal to 0.01(0.1); (b): The TM and TE integrands and summands with (dotted) and without (solid) laser irradiation at $100 \mathrm{~nm}$ separation. The arrow to the left shows how the dark sample $n=0$ term is shifted down at the damping parameter 0.01 .

suring the collective oscillation frequency of the mechanical dipole mode of a BEC near enough to a dielectric substrate for the force to measurably distort the trapping potential. The fractional change in trap frequency is defined as $\gamma_{x} \equiv\left(\omega_{0}-\omega_{x}\right) / \omega_{0}$ in terms of the unperturbed trap frequency, $\omega_{0}$, and $\omega_{x}$, the trap frequency perturbed by the force. In Fig. 9(a) the experimental result ${ }^{5}$ is shown as open squares with error bars. The upper (lower) curve is the theoretical result, without saturation, including (neglecting) the conductivity from the few thermal carriers in the silica wall. We see that also here the neglect of the contribution, to the dielectric function of the silica wall, from the very few thermally excited carriers brings the theoretical result into agreement with experiment. In this geometry, just as in the gold-plate silicon-wafer geometry, the TM modes cause the problems and it is not enough to neglect dissipation to get good agreement between theory and experiment. To include saturation effects we have just modified the zero frequency contribution in analogy with Eq. (5). We note that in this experiment it is enough to have a damping parameter as small as $10^{-10}$ to bring the theoretical result into agreement with experiment.

\section{Summary}

In summary we have proposed that saturation effects are responsible for the discrepancy between theory and experiment in several quite different 


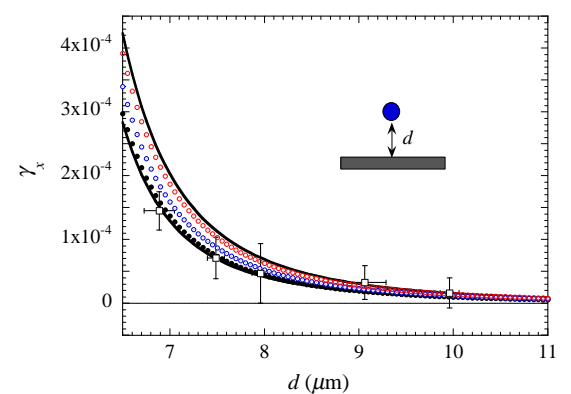

(a)

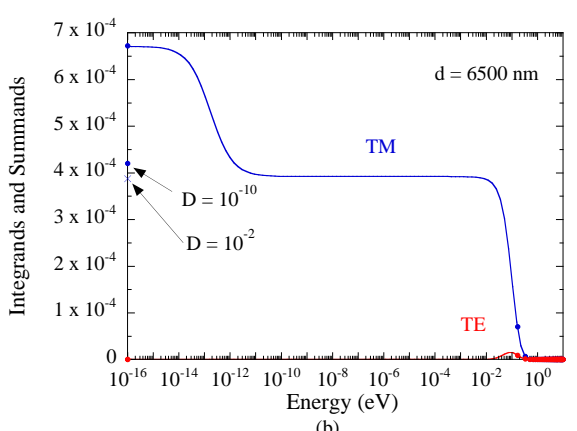

(b)

Fig. 9. (a): Fractional change in trap frequency for a Rb atom near a silica wall versus separation. The open squares are the experimental result. ${ }^{5}$ The upper (lower) curve is the theoretical result including (neglecting) the conductivity from the few thermal carriers in the silica wall. The circles are our present results for the $D$ values $10^{-10}, 10^{-11}$, and $10^{-12}$, respectively, counted from below; (b): The TM and TE integrands and summands at $6500 \mathrm{~nm}$ separation. The arrows to the left shows how the $n=0$ term is shifted down for the damping parameter $10^{-10}$ and $10^{-2}$, respectively.

Casimir geometries. We have demonstrated that the problems may go away in all cases.

\section{Acknowledgments}

The research was sponsored by the VR-contract No:70529001 and support from the VR Linné Centre LiLi-NFM and from CTS is gratefully acknowledged.

\section{References}

1. M. Boström and Bo E. Sernelius, Phys. Rev. Lett 84, 4757 (2000).

2. S. K. Lamoreaux, Phys. Rev. Lett. 78, 5 (1997).

3. R. S. Decca, D. Lopéz, E. Fischbach, G. L. Klimchitskaya, D. E. Krause, and V. M. Mostepanenko, Phys. Rev. D 75, 077101 (2007).

4. F. Chen, G. L. Klimchitskaya, V. M. Mostepanenko, and U. Mohideen, Phys. Rev. B 76, 035338 (2007).

5. J. M. Obrecht, R. J. Wild, M. Antezza, L. P. Pitaevskii, S. Stringari, and E. A. Cornell, Phys. Rev. Lett. 98, 063201 (2007).

6. Bo E. Sernelius, EPL, 8714004 (2009).

7. Bo E. Sernelius, Phys. Rev. A, 80, 043828 (2009).

8. Bo E. Sernelius, Surface Modes in Physics (Wiley-VCH, Berlin, 2001).

9. S. A. Ellingsen, Phys. Rev. E 78, 021120 (2008).

10. Bo E. Sernelius, Phys. Rev. B 71, 235114 (2005).

11. Bo E. Sernelius, Phys. Rev. B 74, 233103 (2006). 